\title{
Article \\ Airborne SAR Radiometric Calibration Based on Improved Sliding Window Integral Method
}

\author{
$\operatorname{Lu~Li}^{1,2,3}$, Fengli Zhang ${ }^{1,2,3, * \mathbb{C}}$, Yun Shao ${ }^{1,2,3}$, Qiufang Wei ${ }^{1}$, Qiqi Huang ${ }^{1,2,3}$ and Yanan Jiao ${ }^{1,2,3}$ \\ 1 Aerospace Information Research Institute, Chinese Academy of Sciences, Beijing 100101, China; \\ lilu192@mails.ucas.ac.cn (L.L.); shaoyun@aircas.ac.cn (Y.S.); weiqf@aircas.ac.cn (Q.W.); \\ huangqiqi20@mails.ucas.ac.cn (Q.H.); jiaoyanan21@mails.ucas.ac.cn (Y.J.) \\ 2 College of Resources and Environment, University of Chinese Academy of Sciences, Beijing 100049, China \\ 3 Laboratory of Target Microwave Properties, Deqing Academy of Satellite Applications, Huzhou 313200, China \\ * Correspondence: zhangfl@aircas.ac.cn; Tel.: +86-10-64838047
}

Citation: Li, L.; Zhang, F.; Shao, Y.; Wei, Q.; Huang, Q.; Jiao, Y. Airborne SAR Radiometric Calibration Based on Improved Sliding Window Integral Method. Sensors 2022, 22, 320. https://doi.org/10.3390/s22010320

Academic Editor: Antonio Pepe

Received: 28 October 2021

Accepted: 29 December 2021

Published: 1 January 2022

Publisher's Note: MDPI stays neutral with regard to jurisdictional claims in published maps and institutional affiliations.

Copyright: (C) 2022 by the authors. Licensee MDPI, Basel, Switzerland. This article is an open access article distributed under the terms and conditions of the Creative Commons Attribution (CC BY) license (https:// creativecommons.org/licenses/by/ $4.0 /)$.

\begin{abstract}
To verify the performance of the high-resolution fully polarimetric synthetic aperture radar (SAR) sensor carried by the Xinzhou 60 remote-sensing aircraft, we used corner reflectors to calibrate the acquired data. The target mechanism in high-resolution SAR images is more complex than it is in low-resolution SAR images, the impact of the point target pointing error on the calibration results is more obvious, and the target echo signal of high-resolution images is more easily affected by speckle noise; thus, more accurate extraction of the point target position and the response energy is required. To solve this problem, this paper introduces image context information and proposes a method to precisely determine the integration region of the corner reflector using sliding windows based on the integral method. The validation indicates that the fully polarimetric SAR sensor on the Xinzhou 60 remote-sensing aircraft can accurately reflect the radiometric characteristics of the ground features and that the integral method can obtain more stable results than the peak method. The sliding window allows the position of the point target to be determined more accurately, and the response energy extracted from the image via the integral method is closer to the theoretical value, which means that the high-resolution SAR system can achieve a higher radiometric calibration accuracy. Additionally, cross-validation reveals that the airborne SAR images have similar quality levels to Sentinel-1A and Gaofen-3 images.
\end{abstract}

Keywords: high-resolution SAR; Xinzhou 60; radiometric calibration; corner reflector; sliding window

\section{Introduction}

Synthetic aperture radar (SAR) has all-day, all-weather Earth observation capabilities and is not subject to interference from clouds, rain, and other bad weather, which is important for applications with strict timeliness requirements, such as emergency disaster monitoring, agricultural monitoring, land resource surveys, topographic surveys, and marine dynamic environment measurement [1]. Many SAR satellites have been launched, and the resolution of spaceborne SAR satellites is continuously improving. Examples include ERS-1/2, JERS-1, and other spaceborne SAR systems, as well as ESA's Envisat ASAR with a $10 \mathrm{~m}$ resolution, Sentinel-1A with a $5 \mathrm{~m}$ resolution, China's HJ-1C with a $5 \mathrm{~m}$ resolution, and other SAR satellites, particularly Italy's COSMO-SkyMed, Canada's Radarsat-2, Germany's TerraSAR-X, and China's Gaofen-3 with a $1 \mathrm{~m}$ resolution. With the increasing resolution of SAR satellites, some applications, such as target detection and target classification, have required the quantification of SAR data [2]. SAR external calibration is the prerequisite for the application of SAR data quantification. There are two types of external calibration methods: one uses point targets, and the other uses distributed targets. In practice, point targets are easier to achieve than large-scale distributed targets with known scattering characteristics and homogeneous stability, and they can achieve the calibration accuracy required by the design [3]. Therefore, the point target calibration 
method is often used to calibrate airborne SAR systems [4]. Additionally, point targets can be applied to the SAR images after calibration to ensure the accuracy of the characteristic backscatter coefficients [5].

Corner reflectors are ground reference instruments that are often used for airborne SAR radiometric calibration. They have a simple structure, stable performance, convenient installation, and low cost, satisfying the requirements of a high radar cross-section (RCS) [6]. In particular, the triangular trihedral corner reflector has a wide beamwidth $\left(\sim 20^{\circ}\right)$ in both the azimuth and elevation dimensions $[7,8]$, and is perhaps the most practical instrument for the calibration of cross-frequency SAR systems [7]. In research on calibration algorithms based on corner reflectors, two different methods-the peak method and the integral method-are often used to combine the RCS and response energy of a point target. Gary et al. reported that after the background clutter and noise are corrected, a straightforward numerical integration of the pixel powers for the corner reflector indicates the best and simplest representation of the RCS. They proposed a simple expression for the relationship between the pixel power in the clutter region and the backscatter coefficient by using the integrated energy for the corner reflector and its RCS [3]. Feng et al. calculated the backscatter coefficients, the system calibration constant, and the system transfer function corresponding to the integral method and the peak method using point targets with known RCSs through actual airborne SAR data and obtained the final images, SCRs, and average backscatter coefficients of typical features to verify the feasibility of using point targets to calibrate the airborne SAR system [4]. Chen et al. conducted a response energy evaluation based on the peak method and the integral method for the corner reflector. They found that both the peak method and the integral method have high calibration accuracies when the image quality is good, and that the integral method can be used first when the image quality is unknown [9]. Zheng et al. found that the integral method has a higher calibration accuracy than the peak method through TerraSAR and airborne SAR calibration experiments based on corner reflectors [10]. In the calculation process, the selection of the center position of the corner reflector is essential for the accurate calculation of the point target response energy. Given the presence of the feature whereby the imaging region of the corner reflector is brighter than the background and occupies many image pixels, Runyi used a region growth algorithm for the automatic extraction of the corner reflector's center in spaceborne SAR images, which uses the "candidate image pixel block" with the largest gray value as the corner reflector's center on the basis of controlling the number of image pixels [11]. Zhao combined the grayscale distribution characteristics of the target pixels to improve the accuracy of the measurement to the subpixel level using the interpolation subdivision algorithm and constructed a subpixel method that reflects the relationship between the grayscale position of the image pixels in the target region and the center of mass position of the target pixels to obtain the accurate target pixel position [12].

To verify the radiometric performance of the high-resolution fully polarimetric SAR system carried by the Xinzhou 60 remote-sensing aircraft, we use the peak method and the integral method to calibrate the actual airborne remote-sensing data using the corner reflectors, and we evaluate the radiometric quality of the airborne SAR images with regard to the SCR, response energy and calibration constants, and accuracy. The SAR system is based on a coherent imaging mechanism [13], and the random interference of ground elements will produce speckle noise, which will not only degrade the image information, affect the visual effect of the image, and interfere with feature classification, target identification, and detection, but also affect the application of SAR image quantification [14]. Furthermore, the background scattered echoes at the deployment position that do not participate in the calculation of the response energy and calibration constants present more detailed information; therefore, the background image is not necessarily completely homogeneous. Those echoes are also superimposed on the point target's echoes, affecting the detection of the point target's response energy, and these echoes are regarded as background clutter. In addition, the current ground reference equipment consists mostly of corner reflectors, which require manual work for angle adjustment, which in turn introduces alignment 
errors between the radar beam and the normal direction of the corner reflector [12]. In other words, the response energy of the point target is the superposition of the echo energy of the point target, background clutter, speckle noise, and other types of echoes [3]. Therefore, the accurate selection of the point target is a key step in ensuring the effectiveness of SAR radiometric calibration. This paper introduces image context information and proposes a method to precisely determine the integration region of the corner reflector using the improved sliding window integral method. We also verified the radiometric calibration effect of the improved method using the Xinzhou airborne SAR data. We cross-validated the airborne SAR images using Sentinel-1A and Gaofen-3 images acquired at approximately the same time to check the quality of the airborne SAR images calibrated using the improved integral method in a large-scale range.

\section{Study Area and Data}

\subsection{Experimental Area}

With the support of China's National Civil Space Infrastructure Land Observation Satellite Common Application Support Platform Project, the Aerospace Information Research Institute of the Chinese Academy of Sciences conducted a comprehensive synergistic experiment involving the acquisition of spaceborne, airborne, and ground remote sensing data in Dongying, Shandong Province, from 10-25 November 2019. We used the Xinzhou 60 remote-sensing aircraft to acquire $\mathrm{P}-,_{-}$-, C-, and X-band high-resolution SAR data and performed simultaneous ground data acquisition. Dongying is located in the northeastern part of Shandong Province, in the delta of the Yellow River estuary, and its central location is approximately $37.69^{\circ} \mathrm{N}, 118.89^{\circ} \mathrm{E}$. The main types of surface coverage are farmland, wetland, waterbody, town, and bare land, and vegetation cover was mainly winter wheat and saline meadow during the experiment. Figure 1 shows the range of the comprehensive experiment in Dongying and the coverage of the multiplatform SAR images. The orange line indicates the coverage area of Sentinel-1A, the blue line indicates the coverage area of Gaofen-3, the green line indicates the airspace area of the Xinzhou 60 remote-sensing aircraft, and the red line indicates the coverage area of the airborne SAR data used in this study. The black triangles indicate the locations of the corner reflectors.

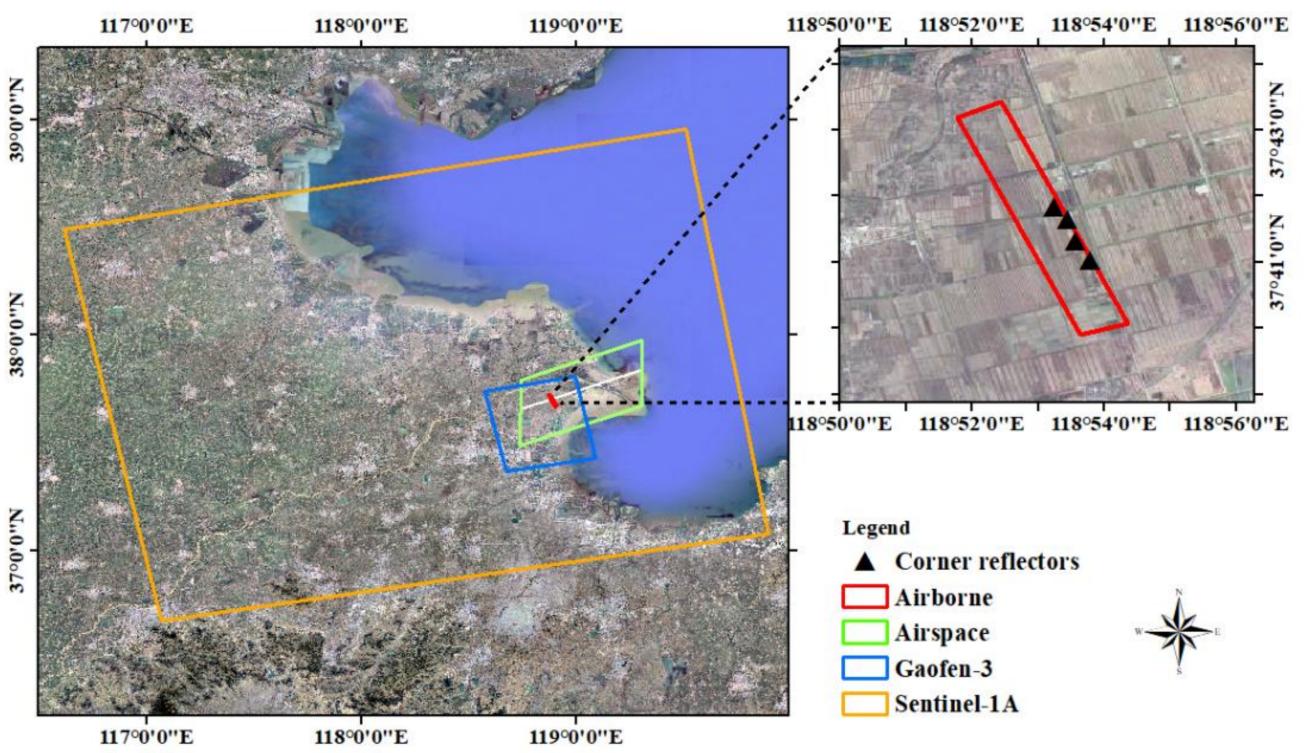

Figure 1. Shandong Dongying comprehensive experimental scope. The orange, blue, and red lines represent the coverage areas of Sentinel-1A, Gaofen 3, and airborne SAR, respectively. The green line indicates the airborne SAR measurement airspace range, and the black triangles represent the corner reflectors. 
The triangular trihedral corner reflector is perhaps the most practical reference for calibrating SAR systems [8]. The peak RCS value for an ideal trihedral is given as [7]:

$$
\sigma_{r e f_{i}}=\frac{4 \pi a^{4}}{3 \lambda^{2}}
$$

where $\sigma_{r e f_{i}}$ represents the peak value for the RCS of the corner reflector, $a$ represents the leg length of the trihedral, and $\lambda$ represents the wavelength of the SAR load. As shown in Figure 2, the leg length of the triangular trihedral corner reflector in the experiment was $700 \mathrm{~mm}$, and the peak RCS under the C-band SAR system was approximately $326.309 \mathrm{~m}^{2}$, i.e., $25.136 \mathrm{dBsm}$. In addition, the accuracy of the corner reflector RCS that we used is $0.2 \mathrm{~dB}$.

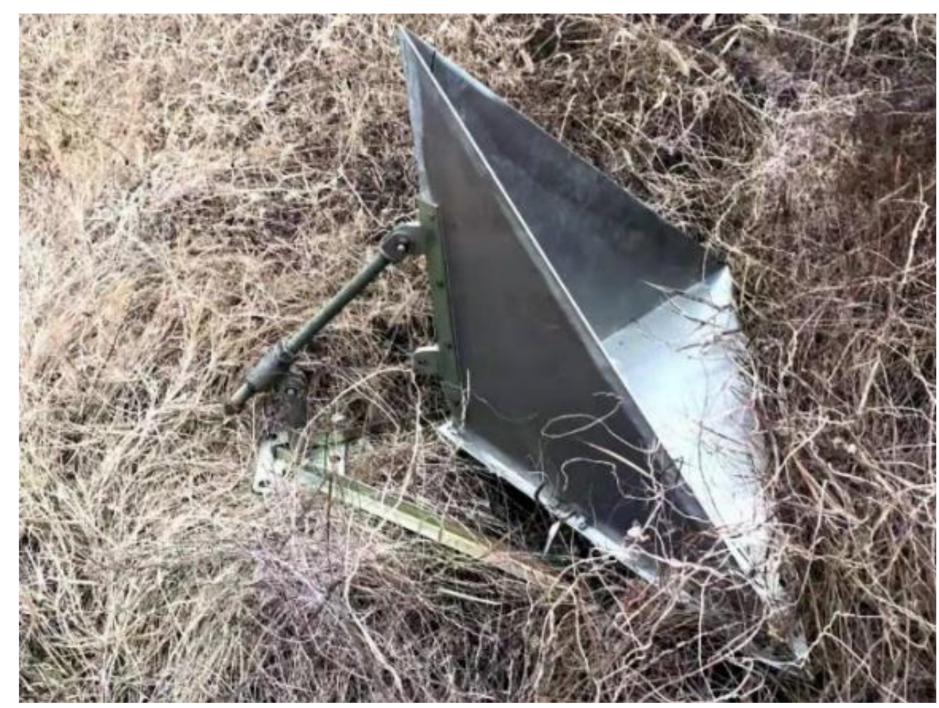

Figure 2. Triangular trihedral corner reflector used in the experiment.

\subsection{Airborne SAR Data}

During the comprehensive experiment, the Xinzhou 60 remote-sensing aircraft-a high-performance, advanced remote-sensing aircraft independently developed by Chinawas used to carry the multi-band SAR sensor of the Aerospace Information Research Institute of the Chinese Academy of Sciences [15] to conduct a large-area synergetic flight observation experiment in Dongying and the surrounding area on 25 November 2019. High-resolution SAR data of the P-, L-, C-, and X-bands were obtained, which can be used in topographic surveying and mapping, environmental protection, disaster monitoring, urban planning, scientific research, national security, etc. The parameters of the sensor are presented in Table 1.

Table 1. Multi-band fully polarimetric SAR parameters.

\begin{tabular}{ccccc}
\hline Band & P & L & C & X \\
\hline Center frequency/GHz & 0.45 & 1.25 & 5.4 & 9.6 \\
Type of polarization & VV\&VH & AHV & AHV & AHV \\
Range resolution $/ \mathrm{m}$ & 3.0 & $3.0-5.0$ & $2.0-3.0$ & $0.3-3.0$ \\
Azimuth resolution $/ \mathrm{m}$ & 3.0 & $3.0-5.0$ & $2.0-3.0$ & $2.0-3.0$ \\
Height accuracy/m & $3-6$ & - & - & $1-3$ \\
Geometrical and & \multicolumn{2}{c}{ Can satisfy feature classification requirements } \\
radiometric accuracy & \multicolumn{7}{c}{} \\
\hline
\end{tabular}

To evaluate the performance of the multi-band high-resolution SAR sensor carried by the Xinzhou 60 remote-sensing aircraft, the C-band was taken as an example, and we 
selected L1-level single-look complex (SLC) images with full polarization, a resolution of $0.5 \mathrm{~m}$, and a flight altitude of $4500 \mathrm{~m}$, which were processed with POS motion compensation, range compression, azimuth focus, and antenna pattern correction. The specific parameters are presented in Table 2. In the airborne simultaneous observation experiment, a total of four triangular trihedral corner reflectors with leg lengths of $700 \mathrm{~mm}$ were placed along the range direction in a uniform farm field in the experimental area, as shown in Figure 3. Because the multi-platform SAR data used for cross-validation all contained VV polarimetric images, the distribution and a local magnification of the corner reflector in the airborne VV polarimetric images are shown in Figures 1 and 3.

Table 2. C-band microwave loading experiment and SLC image parameters.

\begin{tabular}{|c|c|c|c|}
\hline Sensor Parameter & C-SAR & Image Parameters & Content \\
\hline Field of view (FOV) & $35-55^{\circ}$ Left & Radar frequency/GHz & 5.4 \\
\hline Relative altitude/m & 4500 & Type of polarization & Full polarization \\
\hline Resolution/m & 0.5 & Range pixel space/m & 0.20 \\
\hline Bandwidth/km & 3.3 (Antenna) & Azimuth pixel space/m & 0.14 \\
\hline Route Offset $/ \mathrm{km}$ & 3.2 & Center incidence angle $/^{\circ}$ & $53.65^{\circ}$ \\
\hline
\end{tabular}

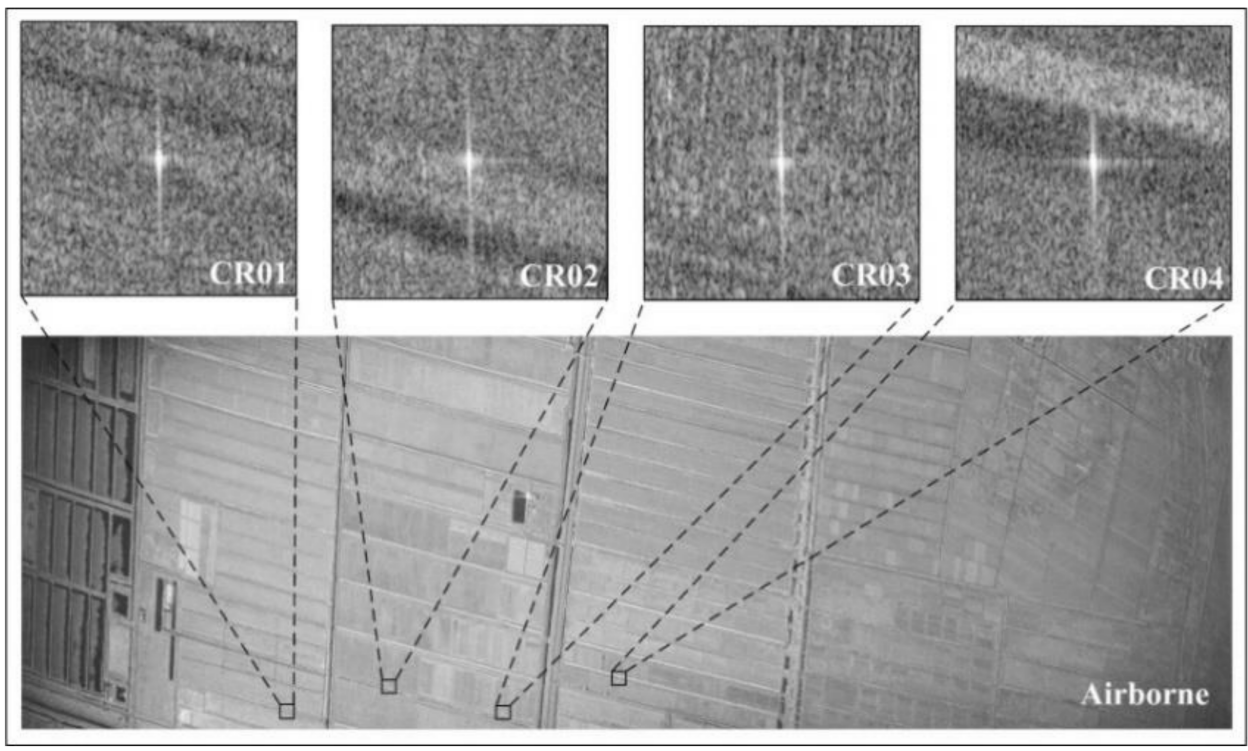

Figure 3. C-band VV polarimetric SAR image and the trihedral corner reflector distribution.

\subsection{Spaceborne SAR Data}

To verify the radiometric calibration results of the high-resolution airborne SAR data on a large scale, Gaofen-3 and Sentinel-1A data with an ascending orbit were acquired at approximately the same time as the airborne images, avoiding daily changes of the surface parameter. The specific parameters are presented in Table 3. The original images were all in SLC format, and the image mode was the quad-polarization strip mode for GaoFen-3 and the interferometric wide swath mode for Sentinel-1A. Both airborne SAR and Gaofen-3 acquired fully polarimetric SAR data during the experiment, whereas Sentinel-1A acquired VV\&VH dual-polarimetric SAR data. Therefore, the VV polarimetric data of the airborne SAR, Gaofen-3, and Sentinel-1A were used as examples to assess the radiometric calibration quality of the airborne SAR images with regard to both direct and cross-validation. The Gaofen-3 image was acquired on 22 November, with a three-day difference from the time of the airborne SAR synchronization experiment. According to local meteorological data, it could be used considering the lack of rain between 22 and 25 November in the study area. Thus, we selected the backscatter coefficients of stable features in spaceborne SAR images for the cross-validation of the airborne SAR data. 
Table 3. Gaofen-3 and Sentinel-1A image parameters.

\begin{tabular}{ccc}
\hline Sensor & Gaofen-3 & Sentinel-1A \\
\hline Imaging Time & 22 November 2019 15:52:24 & 25 November 2019 16:01:48 \\
Band & C & C \\
Center frequency $/ \mathrm{GHz}$ & 5.400 & 5.405 \\
Imaging Mode & QPSI & IW \\
Resolution/m & 8 & 20 \\
Range spacing/m & 2.248 & 2.330 \\
Azimuth spacing/m & 4.849 & 13.932 \\
Type of polarization & Quad & VV\&VH \\
Direction $_{\text {Incidence angle } /{ }^{\circ}}$ & Ascending & Ascending \\
& 25.37 28.02 & $41.49 \sim 45.90$ \\
\hline
\end{tabular}

\section{Methods}

SAR radiometric calibration includes measuring the antenna pattern and measuring the overall transfer function of the radar system (or absolute calibration constant) [16]. When the SAR image is absolutely calibrated, it is necessary to calculate the impulse response energy $\varepsilon_{p}$ of the point target and then obtain the absolute calibration constant $K$ (referred to as the calibration constant) of the stable radar system according to $\varepsilon_{p}$ and the RCS $\sigma$. Finally, we use the calibration constant to absolutely calibrate the SLC data and evaluate the accuracy of the results. The detailed process of SAR calibration is shown in Figure 4.

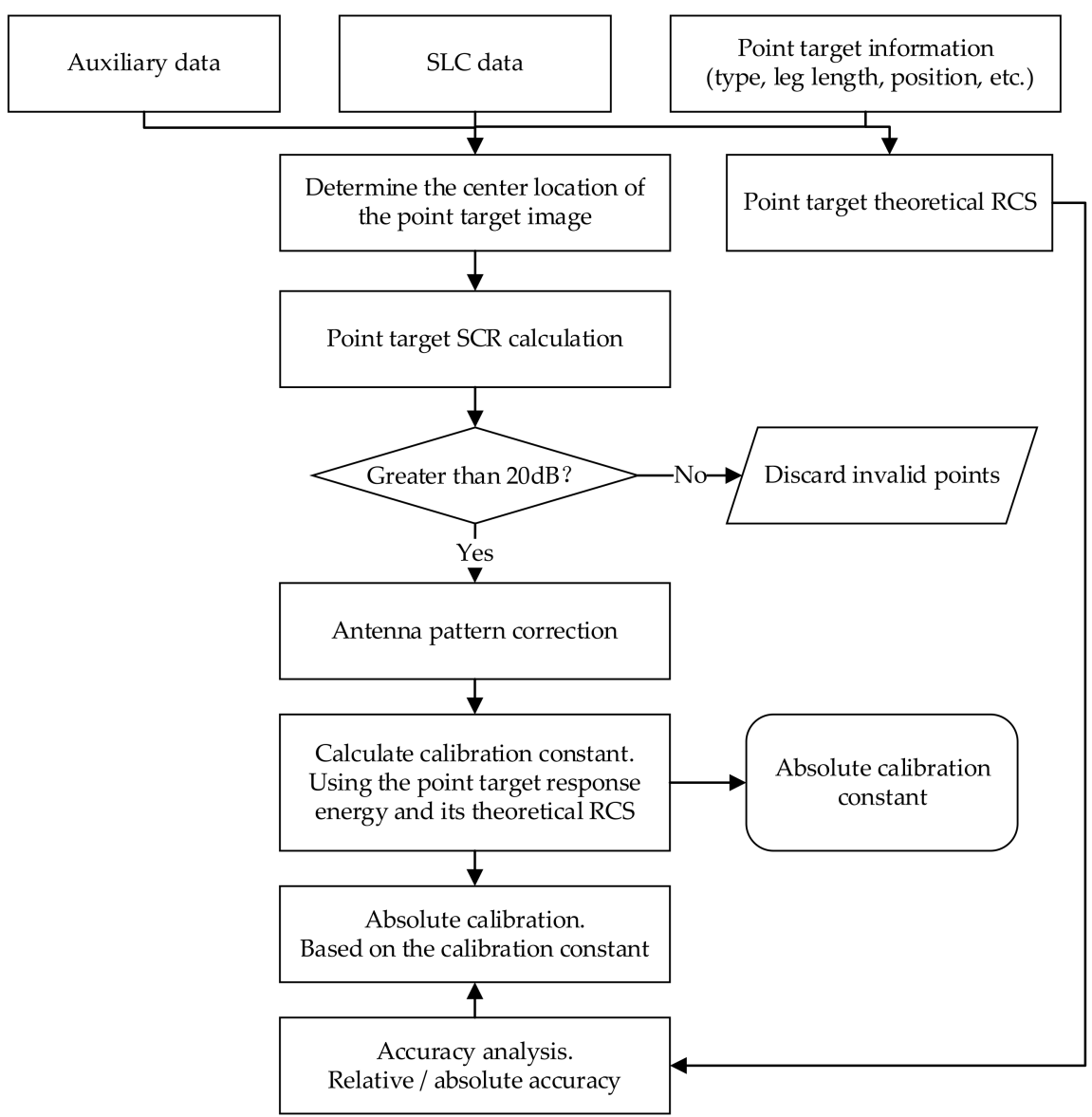

Figure 4. Flowchart of radiometric calibration based on point targets. 


\subsection{Antenna Pattern Correction}

Each SAR's antenna has its antenna pattern. The gain peak is located at the center of the antenna beam and decreases with the increasing angular distance from the center of the beam [17]. The measured power intensity changes according to the beam incidence angle, and when reflected on the SAR image, it manifests as uneven brightness caused by the difference in the incidence angle. Therefore, before the quantitative application of the data, antenna pattern correction is needed to eliminate the amplitude deviation in the range direction of the image caused by the different incidence angles [18]. The basic process of antenna pattern correction based on the corner reflectors is as follows.

(a) Layout corner reflectors: arrange several trihedral corner reflectors of the same leg length at equal intervals along the range direction, accurately determine the coordinates of the corner reflector positions, and adjust the azimuth and elevation angles required for operation.

(b) Antenna pattern fitting: calculate the response energy of each corner reflector in the SAR image and reconstruct the antenna pattern.

(c) Correction coefficient calculation: calculate the ratio of the peak value on the range direction antenna pattern fitting curve to the fitted value of each pixel as the correction coefficient for each position along the range direction.

(d) Image correction: multiply each pixel in the image range direction by the corresponding correction coefficient to eliminate the radiometric deviation caused by the unknown antenna pattern.

\subsection{Point Target Impulse Response Energy Calculation}

When SAR images need to be absolutely calibrated, the key to the determination of the calibration constant is accurately extracting the impulse response energy of the point target. The peak method and the integral method are often used to calculate the impulse response energy of the corner reflector [3,9]. To ensure the validity of the calculation results, it is necessary to measure the SCR of the point target in the actual SAR image before calculating the response energy [7], as indicated by Equation (2). The ratio of the peak power in the target impulse response to the average background clutter power is often used as the SCR, which can be estimated using the area near the point target. Figure 5 shows the specific calculation region for the SCR. When the SCR of the point target is greater than $20 \mathrm{~dB}$, the point target can be considered valid for the subsequent calculation of the response energy and other related calculations [19].

$$
S C R=\frac{\sigma_{p q}^{T}}{\left\langle\sigma_{p q}^{C}\right\rangle}=\frac{\sigma_{p q}^{T} \sin \theta_{i}}{\left\langle\sigma_{p q}^{o}\right\rangle \delta_{a} \delta_{r}},
$$

Here, $\sigma_{p q}^{T}$ represents the point target $\mathrm{RCS},\left\langle\sigma_{p q}^{C}\right\rangle$ represents the average background clutter RCS, $\theta_{i}$ represents the local incidence angle of the point target, and $\delta_{a}$ and $\delta_{r}$ represent the SAR image pixel dimensions in the azimuth and range directions, respectively.

\section{A. Peak estimation method}

The peak method is a function of the peak impulse response of the point target and the equivalent resolution cell area. The use of the peak method requires knowledge of the resolution unit of the SAR system [20]; that is, the $3 \mathrm{~dB}$ antenna main lobe width of the impulse response. Additionally, it is influenced by the system focus. The formula for calculating the impulse response energy of the peak method based on the point target is [21]:

$$
\varepsilon_{r}=D N_{p}^{2} a r \delta_{a} \delta_{r}
$$

where $D N_{p}^{2}$ represents the pixel intensity value of the point target, and $a$ and $r$ are the antenna $3 \mathrm{~dB}$ IRW (impulse response width) in the azimuth and range direction of the 
spread, respectively. In the weighted case, the fair width of the IRW is approximately $20 \%$, namely 1.2 to 1.5 pixels [4,22].

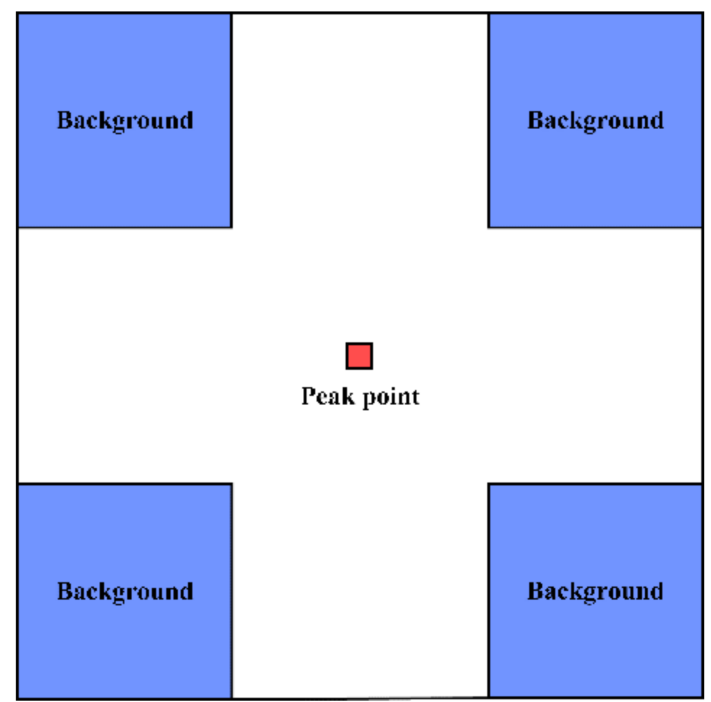

Figure 5. SCR calculation region. The red pixel represents the peak point, and the blue pixels represent the clutter regions.

B. Integral method

The integral method obtains the impulse response energy by integrating the point target impulse response in a specific imaging region. Because the integral method does not need to specify the impulse response of the point target, the calculation is not affected by the system gain, focus, scene, partial coherence of the SAR processor, etc. [3,20]. This feature of the integral method is an advantage over the peak method, and Gary [3] and Cumming [23] have described this idea in detail. In the case of unknown image quality, the integral method is the preferred method for airborne SAR data processing [24]. So far, there is a lot of literature based on the general framework of the integral method and on improving the calibration method for some specific problems, such as the combination of the fit of the sinc antenna pattern and the integral method [18]. The integral response energy of a point target is equal to the difference between the energy of the cross-shaped integration region and the energy of the background calculation region in the integration window of Figure 6 [3].

$$
\varepsilon_{p}=\left(\sum_{i \in A}^{N_{A}} D N_{i}^{2}-\frac{N_{A}}{N_{B}} \sum_{i \in B}^{N_{B}} D N_{i}^{2}\right) \delta_{a} \delta_{r}
$$

Here, $D N_{i}^{2}$ represents the intensity value of the point target pixel, $A$ represents the point target energy integration region (the number of corresponding image pixels is $N_{A}$ ), and $B$ represents the background region (the number of corresponding image pixels is $N_{B}$ ).

The key to the use of the integral method is to accurately and efficiently select the point target integration window to achieve star-ground matching. Therefore, the optimization of the integral method in this paper is for the star-ground matching part of the integration window selection. For the selection of the integration area, the entire point target energy response range must be included, and the background must be relatively uniform to reduce the interference caused by background clutter. The position of the point target is taken as the center, and the $2^{K} \times 2^{K}$ (a natural number $K$ that is greater than 2 is selected according to the actual situation; for our experiment, $K=5$ ) rectangular area is taken as the integration window. In Figure 6, $P$ represents the size of a pixel, area 1 (red area in the figure) is the main lobe area, area 2 (orange) and area 3 (green) are the azimuth side lobe areas, and area 4 (purple) and area 5 (blue) are the range sidelobe areas. 


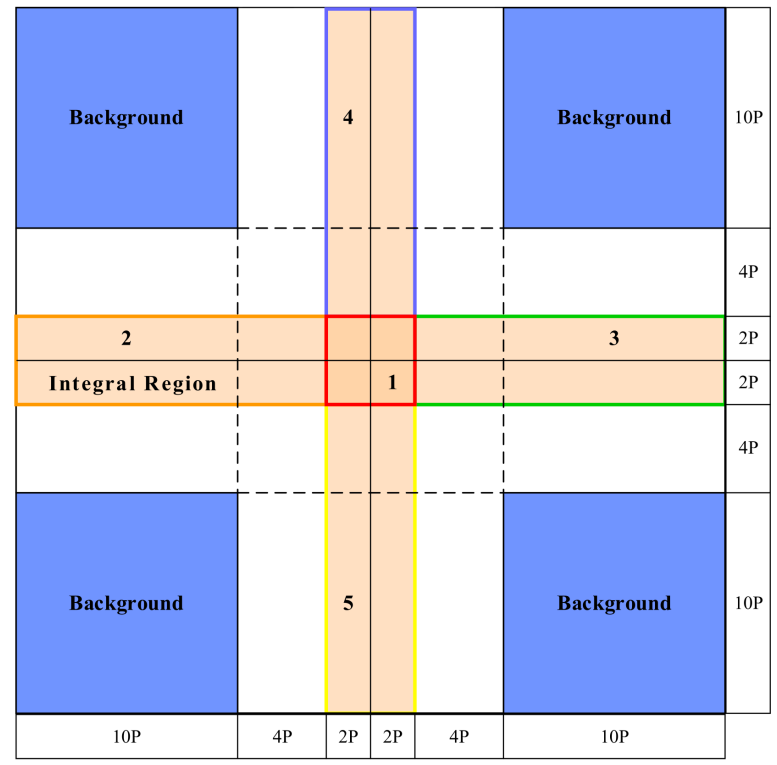

Figure 6. Schematic of the point target response integration area.

\subsection{Optimal Selection of Center Point}

As shown in Figure 6, the determination of the point target's center position is important for accurately selecting the response energy integration window. In an ideal SAR image, the corner reflector appears as a cross-shaped bright spot, and the pixel value is the largest at the spot center, which is the phase center. Therefore, in the traditional method, the maximum center method is often used to determine the center position of the integration area. According to the measured longitude and latitude coordinates of the point target, the initial position on the SAR image is determined by an affine transformation, as indicated by the red pixel in Figure 7. Taking into account the inherent position deviation in the SAR imaging process, a buffer (green line) is set at the initial position of the point target, and the maximum point of the buffer is selected as the center position of the integration window (as indicated by the blue pixel in Figure 7a). However, for the radiometric calibration process of the high-resolution SAR system, the selection of the point target location will inevitably be affected by errors, such as speckle noise [13], background clutter, and alignment errors [12]. This may increase the extraction error of the point target position, affecting the calculation of the integral response energy and the calibration effect. To overcome the difficulties of the maximum center method, we propose the sliding window method to precisely determine the point target location. As shown in Figure $7 \mathrm{~b}$, a buffer area (green line) is taken at the initial position of the point target (red pixel), and then a fixed-sized sliding window (blue box) is applied to slide at a speed of one step, and the sum of the DN values of the window is calculated. Finally, the center point of the sliding window with the largest sum is set as the center point of the integration window (blue pixel in Figure $7 \mathrm{~b}$ ). The calculation process is presented in detail in Algorithm 1. For an ideal point target, the point target integration window obtained via the sliding window method is consistent with that obtained via the maximum center method. In the case of deviations, the sliding window method introduces image context information and uses regional statistics to replace the traditional pixel-based positioning method; thus, it can better correct the point target center selection error. In this way, the cross-shaped energy integration area can be corrected to obtain a more accurate response energy integration window (as indicated by the yellow line in Figure 7b). 


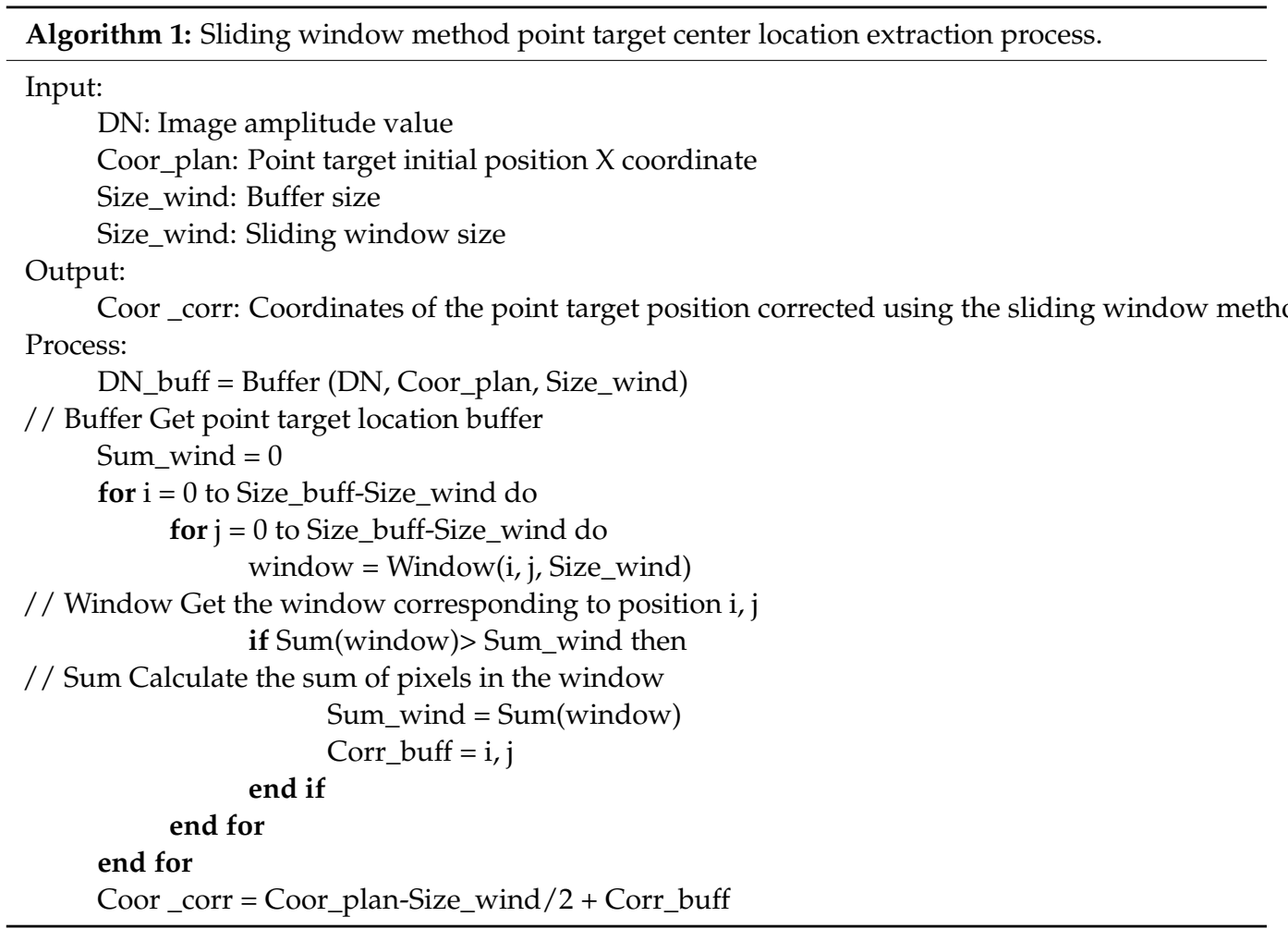

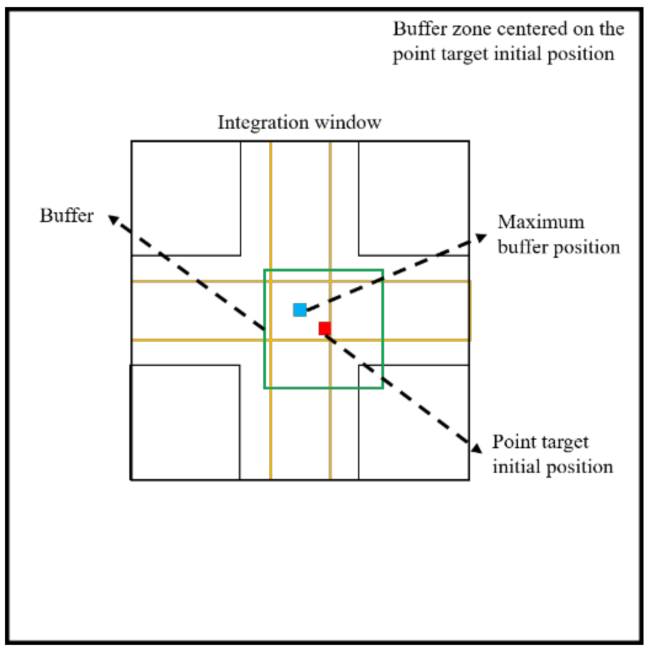

(a)

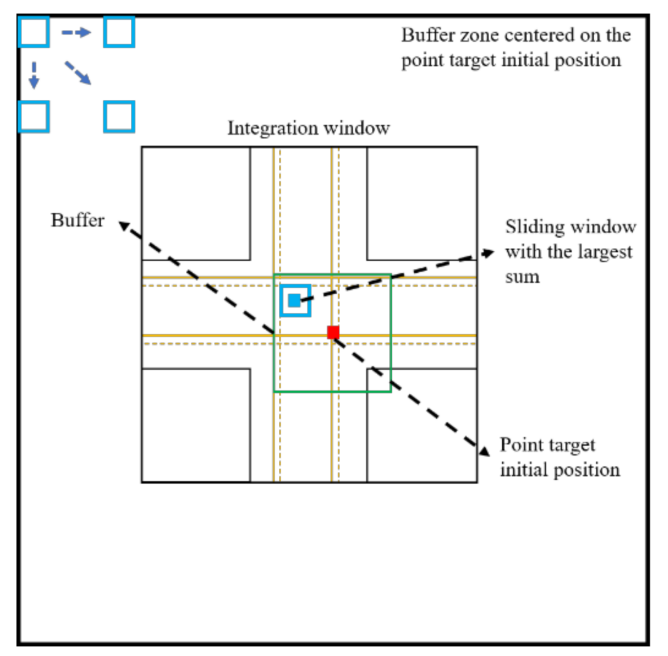

(b)

Figure 7. Schematic of the point target initial positioning method and integration window selection based on (a) the maximum center method and (b) the sliding window method.

\subsection{Calibration Constant Calculation}

When SAR processors need to be absolutely calibrated, it is often necessary to deploy several corner reflectors on the ground to cooperate with the airborne or spaceborne SAR for simultaneous observation. The peak or integral method is then used to extract the corner reflector impulse response energy values and obtain the calibration constants in combination with the known RCS of the corner reflector and its local incidence angle. Specifically, $N$ corner reflectors are placed on the ground, the impulse response energy of corner reflector $i$ is denoted as $\varepsilon_{p_{i}}$, the reference RCS is denoted as $\sigma_{r e f_{i}}$, and the local 
incidence angle is denoted as $\theta_{i}$; then, the calibration constant $K_{i}$ of corner reflector $i$ is given as $[18,25]$ :

$$
K_{i}=\frac{\varepsilon_{p_{i}} \sin \theta_{i}}{\sigma_{r e f_{i}}} .
$$

The average method [26] is often used, which involves taking the average value of the calibration constants of all corner reflectors as the whole scene image's final calibration constant for improving the calculation accuracy $[4,26]$ :

$$
\bar{K}=\frac{1}{N} \sum_{i=1}^{N} K_{i}
$$

After the average value of the calibration constant $\bar{K}$ is obtained, Equation (7) is often used to calculate the backscatter coefficient [4]:

$$
\sigma_{0}=\frac{D N^{2}}{\bar{K}} \cdot \sin \theta
$$

here, $\sigma_{0}$ represents the value of the backscatter coefficient corresponding to the feature, and $D N^{2}$ represents the intensity value of the point target pixel. For SLC data, $D N^{2}$ is calculated using the real part $I$ and imaginary part $Q$; i.e., $D N^{2}=I^{2}+Q^{2}$.

\subsection{Radiometric Calibration Accuracy Analysis}

Radiometric calibration can correct the radiometric distortion caused by the expansion loss effect, non-uniform antenna patterns, gain changes, and speckle noise during SAR imaging processing [17], but the system gain, integrated sidelobe response, etc., may lead to calibration errors. The relative calibration accuracy and absolute calibration accuracy are often used as evaluation indices to describe the accuracy and stability of SAR images.

A. Relative calibration accuracy

Corner reflectors with an equal theoretical RCS should have the same response energy in the SAR image after antenna pattern correction. A certain number of corner reflectors with the same theoretical RCS are placed in the calibration field, and the measured RCS of each corner reflector is calculated and statistically analyzed to obtain the relative calibration accuracy.

$$
\Delta R=\sqrt{\sum_{i=1}^{N} \frac{\left(\widehat{R C S}_{i}-\overline{R C S_{a}}\right)^{2}}{N}},
$$

Here, $\Delta R$ represents the relative calibration accuracy, $\widehat{R C S}_{i}$ represents the measured RCS value of the corner reflector $i$, and $\overline{R C S}_{a}$ represents the average measured RCS value for the $N$ corner reflectors.

\section{B. Absolute calibration accuracy}

Determining the absolute calibration accuracy involves using the calibration constant to calibrate the impulse response of corner reflectors with a known RCS in SAR images to obtain the measured RCS after calibration. The absolute value of the difference between the measured RCS of each corner reflector and the corresponding theoretical RCS is obtained, and the maximum value of the absolute values is taken as the absolute calibration accuracy of the SAR image [18].

$$
\Delta A=\operatorname{Max}\left|\widehat{R C S}_{i}-R C S_{i}\right|,
$$

Here, $\triangle A$ represents the absolute calibration accuracy, and $R C S_{i}$ represents the theoretical RCS value of the corner reflector $i$. With a reduction in $\Delta A$, the difference between the measured and theoretical values is smaller, the absolute calibration accuracy is higher, and the image characteristics are better. 


\section{Results and Discussion}

\subsection{Antenna Pattern Correction Evaluation}

To verify the correction effect of the antenna pattern, the VV polarimetric data were used as an example, and the peak method and the integral method were used to extract the response energy of the corner reflector, as shown in Table 4. The mean energy value obtained via the peak method was $183.609 \mathrm{~dB}$ (with a standard deviation of 0.591 ), and the mean energy value obtained via the integral method was $201.365 \mathrm{~dB}$ (with a standard deviation of 0.551 ). The results indicate that the dispersion and fluctuation of the energy values of the corner reflector are small. As shown in Figure 8, there is no overall tendency for the corner reflector energy to exhibit a significant change in magnitude in the range direction due to the difference in incidence angle offset. Instead, it fluctuates slightly around the mean, indicating that the data are relatively well corrected for the antenna pattern.

Table 4. Validation and analysis of the relative correction of the point target response energy.

\begin{tabular}{ccccc}
\hline \multirow{2}{*}{$\begin{array}{c}\text { Corner } \\
\text { Reflector }\end{array}$} & $\begin{array}{c}\text { Meak Method } \\
\text { Energy/dB }\end{array}$ & Mean/dB & $\begin{array}{c}\text { Standard } \\
\text { Deviation/dB }\end{array}$ & $\begin{array}{c}\text { Measured } \\
\text { Energy/dB }\end{array}$ \\
\hline CR01 & 183.197 & & 200.875 \\
CR02 & 184.308 & 183.609 & 0.591 & 202.059 \\
CR03 & 183.048 & & 200.972 & 2 \\
CR04 & 183.881 & & & 201.552 \\
\hline
\end{tabular}

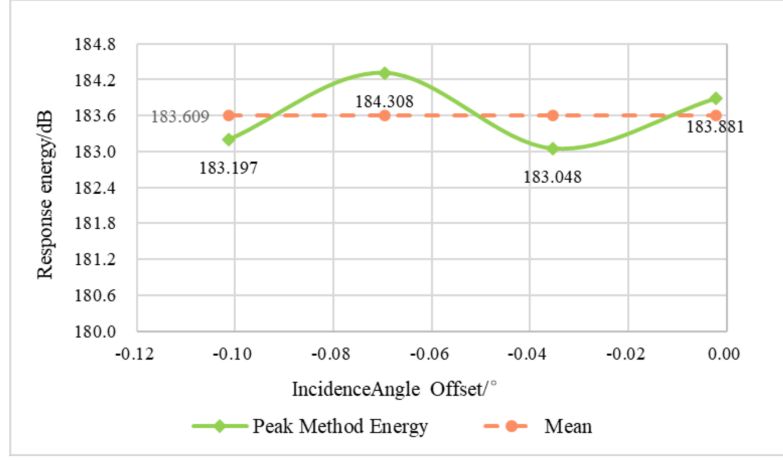

(a)

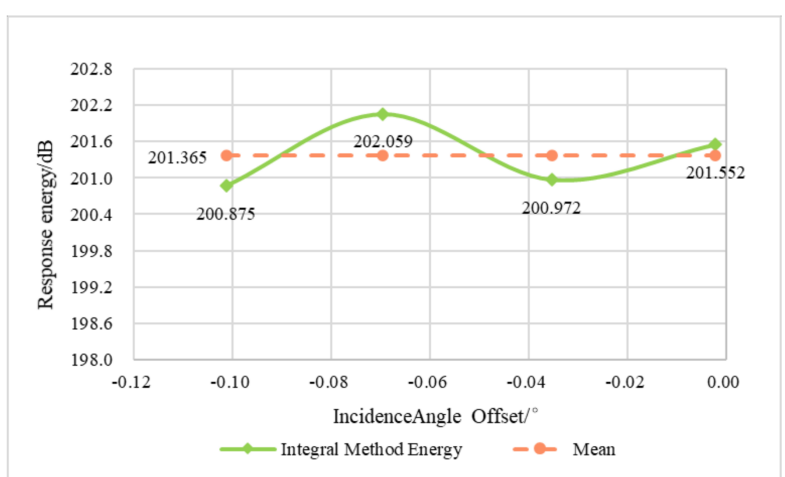

(b)

Figure 8. Verification of the correction results for the corner reflector antenna pattern in the VV polarimetric image: (a) peak method; (b) integral method.

\subsection{Comparative Analysis of Peak Method and Integral Method 4.2.1. Point Target SCR Analysis}

It is necessary to ensure that the imaging of the point target in the SAR image is affected by the background clutter as little as possible; that is, the SCR of the corner reflector $\mathrm{SCR}$ is required to be greater than $20 \mathrm{~dB}$. The VV polarimetric data were also used as an example to verify the SCR results with the corner reflectors. As shown in Table 5, the SCR values of all corner reflectors are greater than $35 \mathrm{~dB}$, indicating that each corner reflector can be considered to be a valid point target in the subsequent calculation.

\subsubsection{Calibration Results and Accuracy Analysis}

The peak response of the corner reflector occupies only one or two pixels in the SAR image, and a fine description of the corner reflector impulse response through interpolation is required. We verified the results of four, eight, and sixteen fast Fourier transform (FFT) interpolations and found that eight-fold FFT interpolation can achieve sufficient accuracy with guaranteed computational efficiency. Therefore, an eight-fold FFT interpolation was 
performed in a $32 \times 32$ neighborhood of the phase center of the corner reflector. The profile lines of the response function in the range and azimuth directions are displayed in a one-dimensional form, as shown in Figure 9.

Table 5. Coordinates and SCR statistics corresponding to each point target in the VV polarimetric image.

\begin{tabular}{cccccc}
\hline $\begin{array}{c}\text { Corner } \\
\text { Reflector }\end{array}$ & Longitude & Latitude & X-Coordinate & Y-Coordinate & SCR/dB \\
\hline CR01 & 118.898 & 37.680 & 5574 & 7905 & 37.768 \\
CR02 & 118.895 & 37.685 & 7680 & 7332 & 37.305 \\
CR03 & 118.893 & 37.690 & 10,064 & 7864 & 37.175 \\
CR04 & 118.889 & 37.695 & 12,494 & 7145 & 40.255 \\
& Mean SCR/dB & & & 38.126 & \\
\hline
\end{tabular}

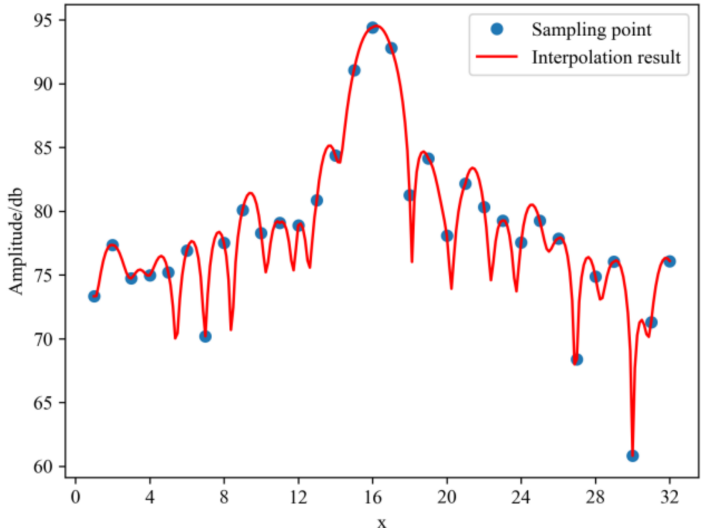

(a)

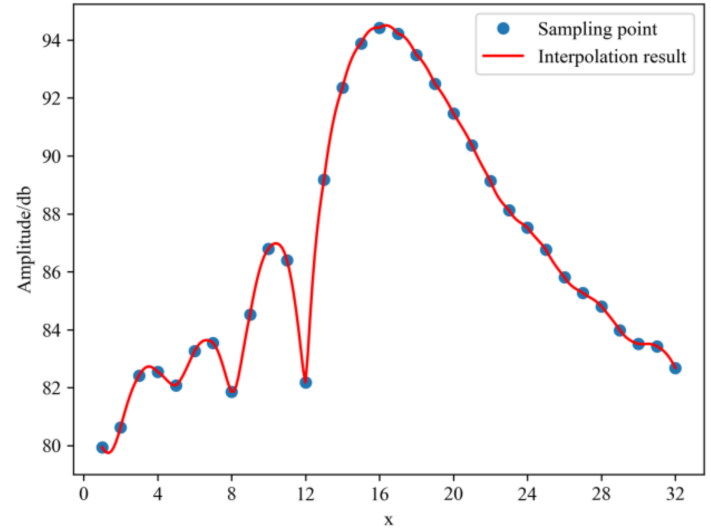

(b)

Figure 9. CR03 one-dimensional profile of the impulse response in the range and azimuth directions in the VV polarimetric image: (a) range direction interpolation; (b) azimuth direction interpolation.

The response energy and calibration constants of each corner reflector are calculated using the peak response and integration region obtained by interpolation. Theoretically, the calibration constants of each corner reflector used in the experiment should be the same in the SAR image after antenna pattern correction. The stability of the results of the two response energy calculation methods is verified by comparing the standard deviations of the calibration constants of the corner reflectors obtained by the two methods. As shown in Table 6, the mean value of the response energy for the peak method is $183.609 \mathrm{~dB}$, and the mean value of the calibration constant obtained by using the theoretical RCS value is $158.472 \mathrm{~dB}$ (with a standard deviation of 0.591 ). The mean value of the integral response energy of the four corner reflectors determined by the integral method is $201.365 \mathrm{~dB}$ and the mean value of the calibration constant is $176.228 \mathrm{~dB}$ (with a standard deviation of 0.551 ). In Figure 10, the calibration constants (green line) corresponding to the peak method exhibit larger fluctuations than the mean value (red line), while the calibration constants (green line) obtained via the integral method exhibit smaller fluctuations than the mean value (red line). The differences between the CR03 calibration constants obtained via the peak and integral methods and their corresponding mean values are 0.561 and $0.392 \mathrm{~dB}$, respectively. In summary, the standard deviation of the calibration constants obtained via the integral method is smaller than that for the peak method, indicating that the calculation results obtained via the integral method are more stable. 
Table 6. Point target response energy and calibration constants obtained by the peak method and the integral method for the VV polarimetric image.

\begin{tabular}{|c|c|c|c|c|c|c|}
\hline \multirow[b]{2}{*}{$\begin{array}{l}\text { Corner } \\
\text { Reflector }\end{array}$} & \multirow[b]{2}{*}{ Length/mm } & \multirow[b]{2}{*}{$\begin{array}{l}\text { Theoretical } \\
\text { RCS/dBsm }\end{array}$} & \multicolumn{2}{|c|}{ Peak Method } & \multicolumn{2}{|c|}{ Integral Method } \\
\hline & & & $\begin{array}{l}\text { Response } \\
\text { Energy/dB }\end{array}$ & Constant/dB & $\begin{array}{l}\text { Response } \\
\text { Energy/dB }\end{array}$ & Constant/dB \\
\hline CR01 & 700 & 25.136 & 183.197 & 158.061 & 200.875 & 175.739 \\
\hline CR02 & 700 & 25.136 & 184.308 & 159.172 & 202.059 & 176.922 \\
\hline CR03 & 700 & 25.136 & 183.048 & 157.911 & 200.972 & 175.836 \\
\hline CR04 & 700 & 25.136 & 183.881 & 158.744 & 201.552 & 176.416 \\
\hline \multicolumn{3}{|c|}{ Mean response energy } & \multicolumn{2}{|c|}{183.609} & \multicolumn{2}{|c|}{201.365} \\
\hline \multicolumn{3}{|c|}{ Mean calibration constants } & \multicolumn{2}{|c|}{158.472} & \multicolumn{2}{|c|}{176.228} \\
\hline \multicolumn{3}{|c|}{ Standard deviation of calibration constants } & \multicolumn{2}{|c|}{0.591} & \multicolumn{2}{|c|}{0.551} \\
\hline
\end{tabular}

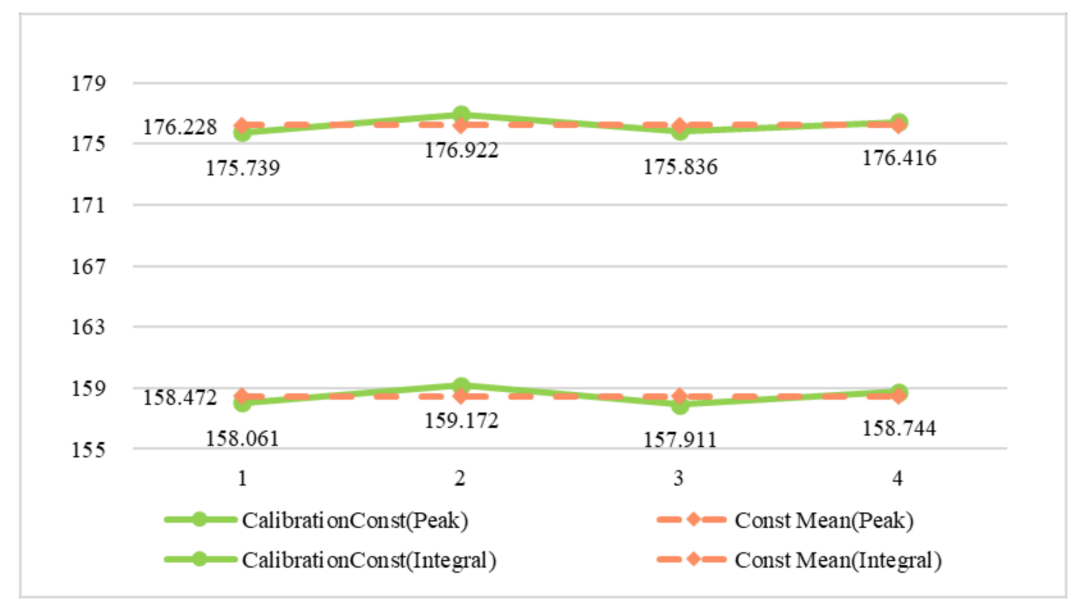

Figure 10. Comparison of the calibration constants and mean values for the VV polarimetric image.

According to the principle of radiometric calibration and accuracy evaluation, the average values of the calibration constants calculated via the peak method and the integral method are used as the calibration constant of the whole image for the absolute calibration and the accuracy evaluation of SAR images, respectively. As shown in Table 7, the relative calibration accuracy of the integral method is $0.551 \mathrm{~dB}$ and the absolute calibration accuracy is $0.668 \mathrm{~dB}$. For the peak method, the relative calibration accuracy is $0.591 \mathrm{~dB}$ and the absolute calibration accuracy is $0.67 \mathrm{~dB}$. The results indicate that the calibration accuracy of the integral method is better than that of the peak method.

Table 7. Point target RCS and accuracy analysis based on the peak method and integral method.

\begin{tabular}{|c|c|c|c|c|c|}
\hline \multirow{2}{*}{$\begin{array}{l}\text { Corner } \\
\text { Reflector }\end{array}$} & \multirow{2}{*}{$\begin{array}{l}\text { Theoretical } \\
\text { RCS/dBsm }\end{array}$} & \multicolumn{2}{|c|}{ Peak Method } & \multicolumn{2}{|c|}{ Integral Method } \\
\hline & & $\begin{array}{l}\text { Measured } \\
\text { RCS/dBsm }\end{array}$ & Difference/dBsm & $\begin{array}{l}\text { Measured } \\
\text { RCS/dBsm }\end{array}$ & Difference/dBsm \\
\hline CR01 & 25.136 & 24.694 & -0.442 & 24.621 & -0.515 \\
\hline CR02 & 25.136 & 25.806 & 0.67 & 25.804 & 0.668 \\
\hline CR03 & 25.136 & 24.545 & -0.591 & 24.717 & -0.419 \\
\hline CR04 & 25.136 & 25.378 & 0.242 & 25.297 & 0.161 \\
\hline \multicolumn{2}{|c|}{ Relative calibration accuracy } & \multicolumn{2}{|c|}{0.591} & \multicolumn{2}{|c|}{0.551} \\
\hline \multicolumn{2}{|c|}{ Absolute calibration accuracy } & \multicolumn{2}{|c|}{0.670} & \multicolumn{2}{|c|}{0.668} \\
\hline
\end{tabular}




\subsection{Improved Method and Results Based on Sliding Windows}

\subsubsection{Center Point Optimization Analysis}

To further improve the calibration accuracy, the positioning of the center point of the corner reflector is optimized by sliding the window, and the SCR of the corner reflector is calculated via the traditional maximum center method and the sliding window method. As shown in Table 8, the image coordinates of CR01 and CR03 differ by one image element in the X direction (azimuth direction), and the image coordinates of CR02 and CR04 are identical. The mean SCR of the maximum center method is $38.126 \mathrm{~dB}$, and the corresponding mean SCR of the sliding window method is $38.130 \mathrm{~dB}$. The mean SCR is improved after optimization, which indicates that the sliding window method can reduce the interference of background clutter.

Table 8. Image coordinates and SCRs of corner reflectors based on the original center position and the improved center position.

\begin{tabular}{ccccccccc}
\hline \multirow{2}{*}{$\begin{array}{c}\text { Corner } \\
\text { Reflector }\end{array}$} & Longitude & Latitude & \multicolumn{3}{c}{ Maximum Center Method } & \multicolumn{3}{c}{ Sliding Window Method } \\
\cline { 4 - 8 } & & & $\mathbf{X}$ & $\mathbf{Y}$ & $\mathbf{S C R} / \mathbf{d B}$ & $\mathbf{X}$ & \multicolumn{1}{c}{$\mathbf{Y}$} & SCR/dB \\
\hline CR01 & 118.898 & 37.680 & 5574 & 7905 & 37.768 & 5573 & 7905 & 37.784 \\
CR02 & 118.895 & 37.685 & 7680 & 7332 & 37.305 & 7680 & 7332 & 37.305 \\
CR03 & 118.893 & 37.690 & 10,064 & 7864 & 37.175 & 10,063 & 7864 & 37.188 \\
CR04 & 118.889 & 37.695 & 12,494 & 7145 & 40.255 & 12,494 & 7145 & 40.255 \\
& Mean SCR & & & 38.126 & & & 38.130 \\
\hline
\end{tabular}

The sliding window is used again for center position selection in the integration region at the subpixel level after FFT interpolation. To clearly compare the selection effects of the integration region before and after optimization, the integration window of the corner reflector is visualized with CR03 as an example and the theoretical cross-shaped integration region of the corner reflector is marked with a solid black line (Figure 11). The center points determined using the sliding window method and the maximum center method are indicated by the white dots in Figure 11. As shown, the corner reflector positions determined via the two methods differ by four subpixels in the X-direction and one subpixel in the Y-direction. Statistical analysis of the cross-shaped integration regions obtained through the two methods indicates that the integral energy obtained through the maximum center method was $216.51 \mathrm{~dB}$, and the integral response energy obtained through the sliding window method was $216.53 \mathrm{~dB}$; thus, the result of the maximum center method was $0.02 \mathrm{~dB}$ lower than that of the sliding window method. Assuming that $145 \mathrm{~dB}$ is the threshold value, the number of pixels in the cross-shaped integration region with values lower than the threshold value obtained via the maximum center method is 972 , while the number of pixels in the integration region obtained via the sliding window method is 818 . Therefore, the number of pixels with a scattering intensity of $<145 \mathrm{~dB}$ in the integration region obtained via the sliding window method is 154 less than that of the maximum center method, which accounts for approximately $1 \%$ of the total number of pixels in the integration region. The energy integration region determined using the maximum center method contains more background pixels; thus, the calculated integral response energy value is small. However, the integration window obtained via the sliding window method can significantly reduce the number of background pixels, which makes the integral response energy extracted from the SAR image closer to the theoretical value of the corner reflector and improves the calculation accuracy. 


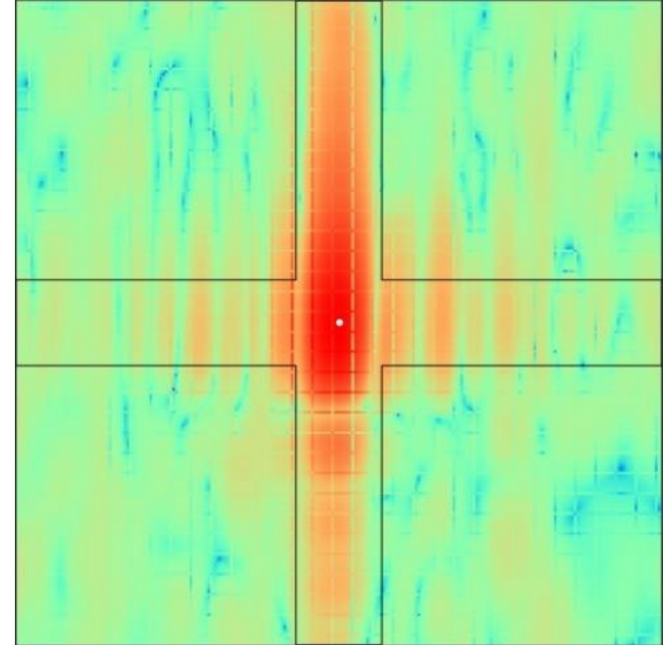

(a)

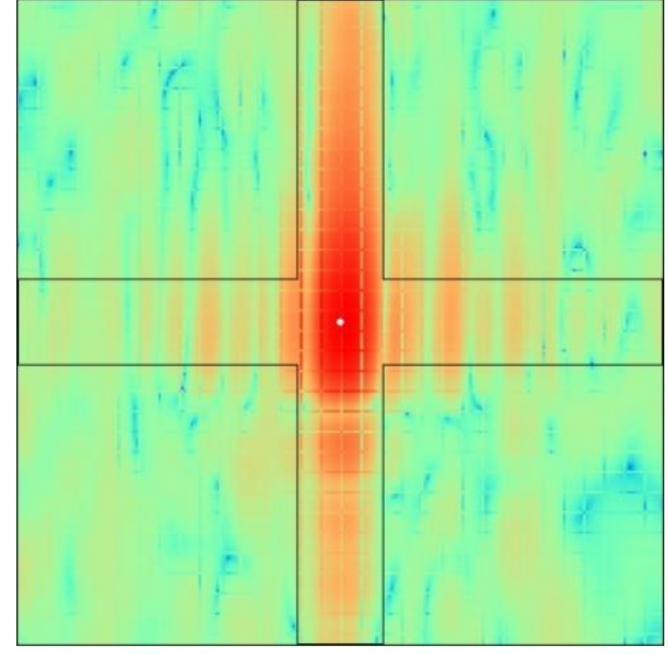

(b)

Figure 11. Integral window of corner reflector CR03 before and after center point position optimization: (a) maximum center method; (b) sliding window method.

\subsubsection{Calibration Results and Accuracy Analysis}

The center point of the integration region is extracted via the methods mentioned above, the response energy of the point target is extracted using the integral method, and the corresponding calibration constants are calculated. As shown in Table 9, the average value of the integral response energy of the point target obtained via the maximum center method is $201.365 \mathrm{~dB}$, and the mean value of the calibration constant calculated by using the theoretical RCS value is $176.228 \mathrm{~dB}$ (with a standard deviation of 0.551 ). The average value of the response energy for the integration regions of the four point targets obtained via the sliding window method is $201.379 \mathrm{~dB}$, and the average value of the calibration constant is $176.242 \mathrm{~dB}$ (with a standard deviation of 0.546 ). When the point target position coordinates, response energy, and calibration constant are employed, the response energy of the corner reflector obtained via the sliding window method is more concentrated than that obtained via the maximum center method. Additionally, the standard deviation of the calibration constants is better for the sliding window method than for the maximum center method, indicating that the integral method improved by the sliding window is more stable and more robust.

Table 9. Integral response energy and calibration constants of the point target for the maximum center method and sliding window method.

\begin{tabular}{|c|c|c|c|c|c|c|}
\hline \multirow{2}{*}{$\begin{array}{l}\text { Corner } \\
\text { Reflector }\end{array}$} & \multirow[b]{2}{*}{ Length/mm } & \multirow{2}{*}{$\begin{array}{l}\text { Theoretical } \\
\text { RCS/dBsm }\end{array}$} & \multicolumn{2}{|c|}{ Maximum Center Method } & \multicolumn{2}{|c|}{ Sliding Window Method } \\
\hline & & & $\begin{array}{l}\text { Response } \\
\text { Energy/dB }\end{array}$ & Constant/dB & $\begin{array}{l}\text { Response } \\
\text { Energy/dB }\end{array}$ & Constant/dB \\
\hline CR01 & 700 & 25.136 & 200.875 & 175.739 & 200.894 & 175.757 \\
\hline CR02 & 700 & 25.136 & 202.059 & 176.922 & 202.068 & 176.932 \\
\hline CR03 & 700 & 25.136 & 200.972 & 175.836 & 200.991 & 175.855 \\
\hline CR04 & 700 & 25.136 & 201.552 & 176.416 & 201.561 & 176.424 \\
\hline \multicolumn{3}{|c|}{ Mean response energy } & \multicolumn{2}{|c|}{201.365} & \multicolumn{2}{|c|}{201.379} \\
\hline \multicolumn{3}{|c|}{ Mean calibration constants } & \multicolumn{2}{|c|}{176.228} & \multicolumn{2}{|c|}{176.242} \\
\hline \multicolumn{3}{|c|}{ Standard deviation of calibration constants } & \multicolumn{2}{|c|}{0.551} & \multicolumn{2}{|c|}{0.546} \\
\hline
\end{tabular}

The average value of the calibration constants obtained via the two integral methods is used as the calibration constant for the entire SAR image to calibrate the SLC data. The measured RCS values of each corner reflector are extracted from the calibration image, and the calibration accuracy is compared with the theoretical RCS value. The relative calibration 
accuracy and absolute calibration accuracy are used as indicators to evaluate the effect of radiometric calibration before and after optimization. As shown in Tables 8 and 10, and Figure 11, the actual integration regions of the four corner reflectors obtained by the sliding window method are significantly corrected, the actual cross-shaped energy integration region overlaps more with the theoretical energy integration region, and the RCS of each corner reflector is improved compared to that of the maximum center method. For the sliding window method, the absolute calibration accuracy is $0.664 \mathrm{~dB}$, and the relative calibration accuracy is $0.546 \mathrm{~dB}$. For the maximum center method, the absolute calibration accuracy is $0.668 \mathrm{~dB}$, and the relative calibration accuracy is $0.551 \mathrm{~dB}$. The calibration accuracies obtained via the sliding window method and the maximum center method both satisfy the radiometric accuracy requirements of the various applications proposed by Ulaby [7] in his report on the scientific requirements for SAR calibration. The relative and absolute calibration accuracies of the improved sliding window integral response energy are better than those obtained via the maximum center method, and the optimized method has more stable and robust calculation results, indicating that the optimized sliding window integral method can significantly improve the quality of airborne SAR calibration.

Table 10. Point target RCS and accuracy analysis for the maximum center method and sliding window method.

\begin{tabular}{|c|c|c|c|c|c|}
\hline \multirow{2}{*}{$\begin{array}{l}\text { Corner } \\
\text { Reflector }\end{array}$} & \multirow{2}{*}{$\begin{array}{l}\text { Theoretical } \\
\text { RCS/dBsm }\end{array}$} & \multicolumn{2}{|c|}{ Maximum Center Method } & \multicolumn{2}{|c|}{ Sliding Window Method } \\
\hline & & $\begin{array}{l}\text { Measured } \\
\text { RCS/dBsm }\end{array}$ & Difference/dBsm & $\begin{array}{l}\text { Measured } \\
\text { RCS/dBsm }\end{array}$ & Difference/dBsm \\
\hline CR01 & 25.136 & 24.621 & -0.515 & 24.624 & -0.512 \\
\hline CR02 & 25.136 & 25.804 & 0.668 & 25.800 & 0.664 \\
\hline CR03 & 25.136 & 24.717 & -0.419 & 24.723 & -0.413 \\
\hline CR04 & 25.136 & 25.297 & 0.161 & 25.293 & 0.157 \\
\hline \multicolumn{2}{|c|}{ Relative calibration accuracy } & \multicolumn{2}{|c|}{0.551} & \multicolumn{2}{|c|}{0.546} \\
\hline \multicolumn{2}{|c|}{ Absolute calibration accuracy } & \multicolumn{2}{|c|}{0.668} & \multicolumn{2}{|c|}{0.664} \\
\hline
\end{tabular}

\subsection{Cross-Validation Analysis}

To verify the radiometric calibration results of high-resolution airborne SAR data based on the integral method improved by the sliding window on a large scale, the optimized integral method was applied to the calibration of airborne $\mathrm{HH}$ and VV polarimetric images from the experiment, and the Gaofen-3 and Sentinel-1A images acquired at approximately the same time as the airborne SAR data were used for cross-validation. Taking into account the 3-day difference between the Gaofen-3 data and the time of the airborne SAR synchronization experiments, differences in precipitation and wind speed may lead to changes in the intensity of the backscatter of large, distributed features, such as farmlands and ponds. Additionally, the spatial resolution of Sentinel-1A is low, and small areas or independent features, such as roads, are difficult to identify via visual interpretation. To ensure the reliability of the validation results, the cross-validation selects features whose backscatter coefficients do not easily change over time for comparison; that is, the comparison of the backscatter coefficients in the building area. The backscatter coefficients of buildings in different images cannot be directly compared due to differences in the incidence angles of the SAR data from the three platforms. Therefore, according to reference [27], the experiments were performed using a theoretical approach based on Lambert's law for optics as proposed by Ulaby [28] for the radiometric normalization of the multiplatform data, and the normalized backscatter coefficients with a reference incidence angle of $35^{\circ}$ were obtained and compared. As shown in Figure 12 and Table 11, the average value of the backscatter coefficient of the building area for the sensors under $\mathrm{HH}$ polarization is $1.25 \mathrm{~dB}$ (with a standard deviation of 0.03 ), while the average value under VV polarization is $0.74 \mathrm{~dB}$ (with a standard deviation of 0.01 ). The backscatter coefficient of the building area under VV polarimetric data is slightly smaller than that under $\mathrm{HH}$ polarimetric data, 
which is consistent with the regular imaging of the fully polarimetric SAR system. The maximum difference between the building area backscatter coefficients of the airborne SAR and spaceborne SAR (Gaofen-3) images in the $\mathrm{HH}$ polarimetric data is $0.05 \mathrm{~dB}$, and the maximum difference between those of the airborne SAR and spaceborne SAR (Sentinel-1A) images in the VV polarimetric data is $0.02 \mathrm{~dB}$. In summary, the airborne SAR calibration results are consistent with the backscatter coefficients of the building area in the spaceborne SAR data, indicating that the airborne SAR data have high reliability and can effectively reflect the radiometric characteristics of the ground features.

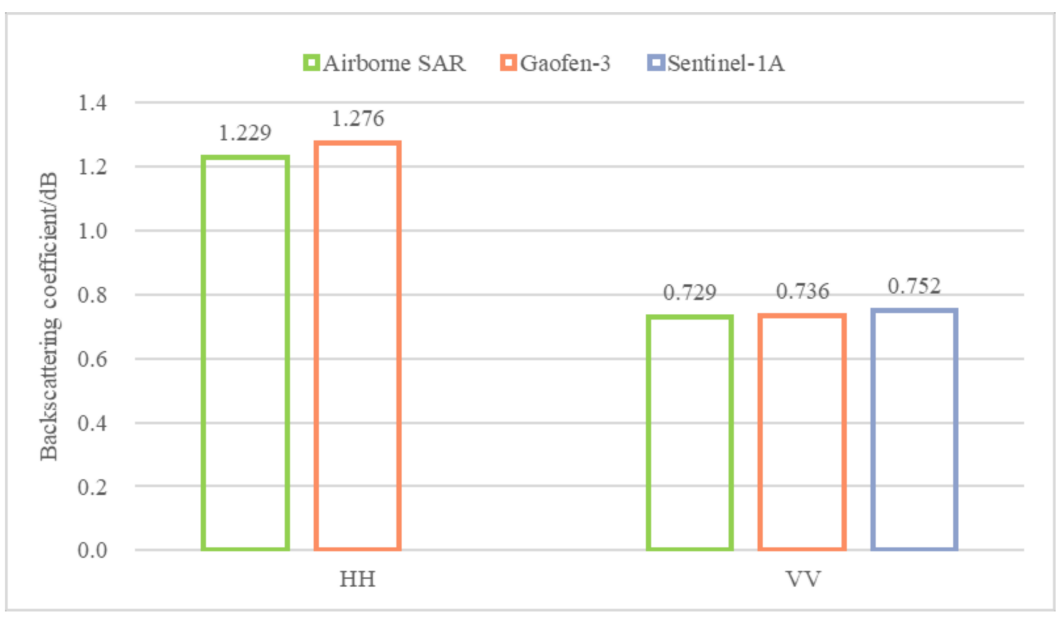

Figure 12. Comparison of normalized backscatter coefficients in the building area obtained with different sensors.

Table 11. Statistical results for the backscatter coefficients in the building area obtained with different sensors.

\begin{tabular}{ccc}
\hline Sensors & HH Polarization & VV Polarization \\
\hline Airborne SAR & 1.229 & 0.729 \\
Gaofen-3 & 1.276 & 0.736 \\
Sentinel-1A & - & 0.752 \\
\hline
\end{tabular}

\section{Conclusions}

We used the peak method and the integral method combined with ground corner reflectors to calibrate airborne SAR data and found that the high-resolution SAR system carried by the Xinzhou 60 remote-sensing aircraft can effectively reflect the radiometric characteristics of the ground features. To address many factors that influence the imaging of the high-resolution SAR system, this paper introduced image context information and proposed a method to precisely determine the integration region of the corner reflector using sliding windows based on the integral method. We used high-resolution airborne SAR data acquired by the Xinzhou 60 remote-sensing aircraft for verification. The results indicated that the sliding window allows the position of the point target on the image to be determined more accurately, and the response energy extracted from the image based on the integral method is closer to the theoretical value of the point target, which enables the high-resolution SAR system to achieve higher calibration accuracy. Additionally, crossvalidation of the calibrated airborne SAR images with multi-platform spaceborne SAR images acquired at approximately the same time indicated that the airborne SAR images had a similar quality level to the Sentinel-1A and Gaofen-3 images, and have the data conditions for quantitative research. 


\begin{abstract}
Author Contributions: L.L. undertook the data curation, designed the methodology, conducted the formal analysis, and wrote the manuscript; F.Z. provided the resources, supervised the study, and revised the manuscript; Y.S. provided the resources, supervised the study, and gave comments and suggestions. Q.W. undertook the data curation and provided the resources. Q.H. and Y.J. assisted L.L. in designing the experiments and validating the results. All authors have read and agreed to the published version of the manuscript.
\end{abstract}

Funding: This work was supported by the Common Application Support Platform for Land Observation Satellites of China's Civil Space Infrastructure (CASPLOS_CCSI) and the China high-resolution earth observation system (21-Y20B01-9003-19/22).

Institutional Review Board Statement: Not applicable.

Informed Consent Statement: Not applicable.

Data Availability Statement: Not applicable.

Acknowledgments: Our thanks for the support with acquiring the data to all members of the Central China Comprehensive Experimental Group of the Common Application Support Platform for Land Observation Satellites of China's Civil Space Infrastructure Project in 2019. In addition, the author would like to thank the anonymous reviewers for their constructive comments and suggestions.

Conflicts of Interest: The authors declares that there is no conflict of interest regarding the publication of this paper.

\title{
References
}

1. Moreira, A. A golden age for spaceborne SAR systems. In Proceedings of the 2014 20th International Conference on Microwaves, Radar and Wireless Communications (MIKON), Gdansk, Poland, 16-18 June 2014; pp. 1-4.

2. El-Darymli, K.; McGuire, P.; Gill, E.; Power, D.; Moloney, C. Understanding the significance of radiometric calibration for synthetic aperture radar imagery. In Proceedings of the 2014 IEEE 27th Canadian Conference on Electrical and Computer Engineering (CCECE), Toronto, ON, Canada, 4-7 May 2014; pp. 1-6.

3. Gray, A.L.; Vachon, P.W.; Livingstone, C.E.; Lukowski, T.I. Synthetic aperture radar calibration using reference reflectors. IEEE Trans. Geosci. Remote Sens. 1990, 28, 374-383. [CrossRef]

4. Zongmin, F.; Lei, H.; Zhihua, T.; Jiuli, L.; Liangbo, Z. Airborne SAR radiometric calibration using point targets. IOP Conf. Ser. Earth Environ. Sci. 2014, 17, 012186. [CrossRef]

5. Döring, B.J.; Looser, P.; Jirousek, M.; Schwerdt, M. Point target correction coefficients for absolute SAR calibration. In Proceedings of the 2011 IEEE International Instrumentation and Measurement Technology Conference, Hangzhou, China, 10-12 May 2011; pp. 1-6.

6. Zhou, Y.; Li, C.; Ma, L.; Yang, M.Y.; Liu, Q. Improved trihedral corner reflector for high-precision SAR calibration and validation. In Proceedings of the 2014 IEEE Geoscience and Remote Sensing Symposium, Quebec City, QC, Canada, 13-18 July 2014; pp. $454-457$.

7. Freeman, A. Sar Calibration: An Overview. IEEE Trans. Geosci. Remote Sens. 1992, 30, 1107-1121. [CrossRef]

8. Gisinger, C.; Schubert, A.; Breit, H.; Garthwaite, M.; Balss, U.; Willberg, M.; Small, D.; Eineder, M.; Miranda, N. In-Depth Verification of Sentinel-1 and TerraSAR-X Geolocation Accuracy Using the Australian Corner Reflector Array. IEEE Trans. Geosci. Remote Sens. 2021, 59, 1154-1181. [CrossRef]

9. Chen, J.; Zhang, B.; Wang, C.; Lei, W.; Wu, F. Comparative Study on the Applicability of Two Corner Reflector based Radiometric Calibration Methods for High Resolution Airborne SAR Image. Remote Sens. Technol. Appl. 2015, 30, 677-683.

10. Zheng, C.; Huang, L.; Chen, Q. Accuracy of Airborne SAR Radiometric Calibration with Point Target. Remote Sens. Inf. 2015, 30, 14-19.

11. Run, Y. On-Orbit Geometric Calibration of GF-3 Satellite and Joint-Positioning of GF-3 and GF-2 Satellite Images. Master's Dissertation, WuHan University, Wuhan, China, 2017.

12. Zhao, R. Research on Model and Method of Geometric Calibration for Space-Borne SAR. Ph.D. Dissertation, Liaoning University of Engineering and Technology, Jinzhou, China, 2017.

13. Liu, S.J.; Wu, G.Q.; Zhang, X.Z.; Zhang, K.; Wang, P.; Li, Y.M. SAR despeckling via classification-based nonlocal and local sparse representation. Neurocomputing 2017, 219, 174-185. [CrossRef]

14. Choi, H.; Jeong, J. Speckle Noise Reduction Technique for SAR Images Using Statistical Characteristics of Speckle Noise and Discrete Wavelet Transform. Remote Sens. 2019, 11, 1184. [CrossRef]

15. Zhu, J.; Ding, C.; Pan, J.; Zhou, L.; Yang, H. Research on System Integration of Airborne Remote Sensing Sensors and MA60 Flight Platform. In Proceedings of the 4th Annual Conference on High Resolution Earth Observation, Wuhan, China, 17 September 2017.

16. Zhang, G.; Jiang, Y.; Li, L.; Deng, M.; Zhao, R. Research progress of high-resolution optical/SAR satellite geometric radiometric calibration. Acta Geod. Cartogr. Sin. 2019, 48, 1604-1623. 
17. Larson, R.W.; Jackson, P.L.; Kasischke, E.S. A digital calibration method for synthetic aperture radar systems. IEEE Trans. Geosci. Remote Sens. 1988, 26, 753-763. [CrossRef]

18. Yang, S.; Xu, Z.; Cheng, C. Method of airborne SAR radiation calibration based on point target. In Proceedings of the International Archives of the Photogrammetry, Remote Sensing and Spatial Information Sciences-ISPRS Archives, Online, 14-20 June 2020.

19. Touzi, R.; Hawkins, R.K.; Cote, S. High-Precision Assessment and Calibration of Polarimetric RADARSAT-2 SAR Using Transponder Measurements. IEEE Trans. Geosci. Remote Sens. 2013, 51, 487-503. [CrossRef]

20. Ulander, I.M.H. Accuracy of using point targets for SAR calibration. IEEE Trans. Aerosp. Electron. Syst. 1991, 27, 139-148. [CrossRef]

21. Praveen, T.N.; Raju, M.V.; Vishwanath, B.D.; Meghana, P.; Manjula, T.R.; Raju, G. Absolute radiometric calibration of RISAT-1 SAR image using peak method. In Proceedings of the 2018 3rd IEEE International Conference on Recent Trends in Electronics, Information \& Communication Technology (RTEICT), Bangalore, India, 18-19 May 2018; pp. 456-460.

22. Zink, M.; Bamler, R. X-SAR radiometric calibration and data quality. IEEE Trans. Geosci. Remote Sens. 1995, 33, 840-847. [CrossRef]

23. Cumming, I.G.; Wong, F.H. Digital Processing of Synthetic Aperture Radar Data Algorithm and Implementation. Artech House 2005, 1, 108-110.

24. Oliver, C.; Quegan, S. Understanding Synthetic Aperture Radar Images; Artech House: Boston, MA, USA; London, UK, 1998.

25. Yuan, L.; Li, Z.; Ge, J.; Jiang, K. Research on Approach of SAR Radiometric Calibration Using Point Target. Radio Eng. 2009, 39, 25-28.

26. Wang, Q.; Zeng, Q.; Jiao, J.; Zhou, X.; Liang, C.; Gao, S. Determination of vertical antenna pattern of high-resolution airborne SAR and radiometric calibration. In Proceedings of the 2012 IEEE International Geoscience and Remote Sensing Symposium, Munich, Germany, 22-27 July 2012; pp. 4022-4025.

27. Bai, X.; He, B.; Li, X.; Zeng, J.; Wang, X.; Wang, Z.; Zeng, Y.; Su, Z. First assessment of Sentinel-1A data for surface soil moisture estimations using a coupled water cloud model and advanced integral equation model over the Tibetan Plateau. Remote Sens. 2017, 9, 714. [CrossRef]

28. Ulaby, F.T.; Moore, R.K.; Fung, A.K. Microwave Remote Sensing: Active and Passive, Volume II: Radar Remote Sensing and Surface Scattering and Emission Theory; Artech House Publishers: Norwood, MA, USA, 1986. 